\title{
Cardiac Lipoma of the Interventricular Septum
}

\author{
$\underline{\text { Rodrigo Maia Alves }}^{1}$, Cristina Gamboa ${ }^{2}$ \\ ${ }^{1}$ Medicine Department, Internal Medicine Service, Hospital Sousa Martins, Guarda, Portugal \\ ${ }^{2}$ Medicine Department, Cardiology Service, Hospital Sousa Martins, Guarda, Portugal
}

Received: 07/05/2020

Accepted: $13 / 05 / 2020$

Published: 10/06/2020

How to cite this article: Maia Alves R, Gamboa C. Cardiac lipoma of the interventricular septum. EJCRIM 2020;7: doi:10.12890/2020_001685.

Conflicts of Interests: The Authors declare that there are no competing interests.

Acknowledgements: We would like to thank Dr Anabela Dinis Vargas and Dr Rita Rodrigues for their contribution to image acquisition and processing.

This article is licensed under a Commons Attribution Non-Commercial 4.0 License

\section{ABSTRACT}

Cardiac lipomas are rare benign primary neoplasms of the heart, usually found incidentally, that can become symptomatic depending on their size and location. We report the case of a 61-year-old man presenting with chest pain and elevated troponin and a normal EKG and D-dimers. A transthoracic echocardiogram revealed an intracardiac mass attached to the interventricular septum protruding to the left ventricle, later confirmed to be a lipomatous mass consistent with a cardiac lipoma on cardiac magnetic resonance imaging. Due to the mass characteristics and favourable evolution, it was decided not to excise the tumour, and the patient remains asymptomatic after a 4-year follow-up period.

\section{LEARNING POINTS}

- Cardiac lipomas are rare benign tumours, usually found incidentally, although they can cause a wide array of symptoms depending on their size and location.

- Cardiac magnetic resonance imaging is the most accurate diagnostic method to characterize lipomatous cardiac masses and to distinguish lipomas from liposarcomas.

- Surgical excision is the mainstay of the treatment when feasible, although small asymptomatic lipomas can be managed with a vigilant non-invasive approach.

\section{KEYWORDS}

Cardiac lipoma, primary neoplasms of the heart, cardiac magnetic resonance imaging

\section{CASE DESCRIPTION}

A 61-year-old man, recently diagnosed with rheumatoid arthritis, presented to the emergency department with a first episode of constant precordial pain, radiating to his right arm and dorsal region, that worsened with deep inspiration, for the previous 24 hours. The pain was unrelated to physical activity and increased progressively. He also complained of accompanying sweats, denying any dyspnoea, cough or fever. As chronic medication, he was taking $5 \mathrm{mg}$ of prednisolone and $50 \mathrm{mg}$ of diclofenac per day. Vital signs were normal, and no heart murmurs or fremitus were present, with normal breath sounds and an unremarkable general examination.

The electrocardiogram showed sinus rhythm, a heart rate of $88 \mathrm{bpm}$ and no signs of ischaemia. On chest x-ray, a slightly increased cardiothoracic ratio was noted with no other significant changes. The patient was referred for a cardiology consultation where a transthoracic echocardiogram (TTE) revealed a solid mass in the left ventricle (LV), arising from the distal segment of the interventricular septum (IVS) and posterior wall (Fig. 1). There was no chamber enlargement or hypertrophy and no significant valve defects, with preserved global systolic and diastolic function. 

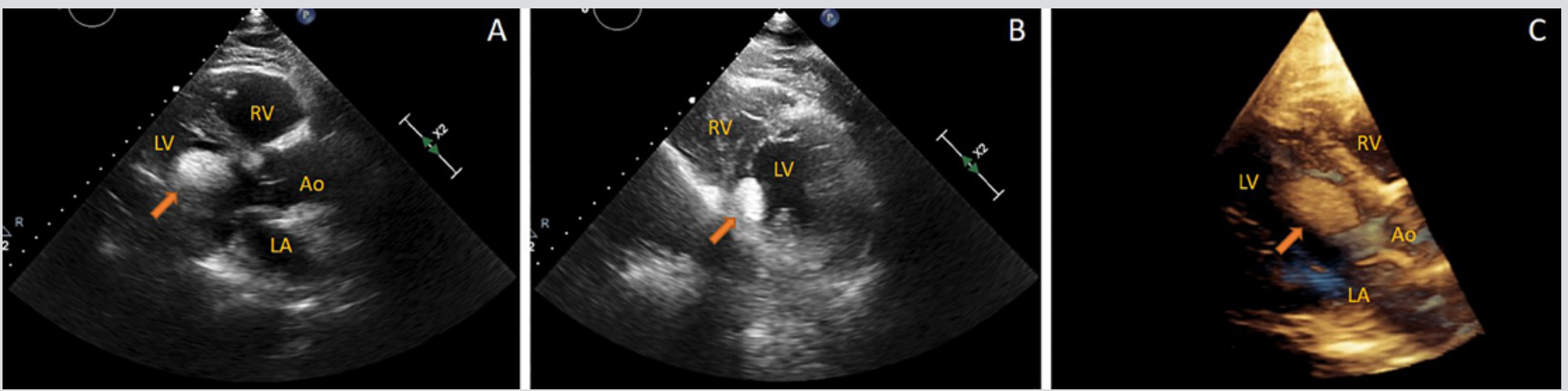

Figure 1. (A) TTE: parasternal long-axis view; (B) parasternal short-axis left ventricular mid view; (C) 3D TTE long-axis view. The arrows indicate the lipoma Ao, aorta; $L A$, left atrium; $L V$, left ventricle; $R V$, right ventricle

Cardiac enzymes were elevated, namely CK was $400 \mathrm{U} / \mathrm{I}$ (normal values: 30-200), CK-MB $43.9 \mathrm{ng} / \mathrm{ml}$ (normal values: 0.0-4.0), troponin I 547.9 pg/ml (normal values: $<34.20$ pg/ml), BNP 601.2 pg/ml (normal values: 0.0-100.0), C-reactive protein 9.05 mg/dl (normal values: $<0.50$ ) and normal age-adjusted D-dimer. Arterial blood gas only showed mild respiratory alkalosis. The pain gradually resolved, and cardiac enzymes measured again 3 hours later displayed a marked decrease, with CK 93 U/I, CK-MB 27.9 ng/ml and troponin I 71.1 pg/ml, normalizing in the first 24 hours.

The patient was then admitted for monitoring and further investigation, and coronary computed tomography angiography (cCTA) performed to access coronary and cardiac anatomy, excluded significant atherosclerotic lesions and confirmed the presence of the mass in continuity with the IVS suggesting lipomatous characteristics (Fig. 2).

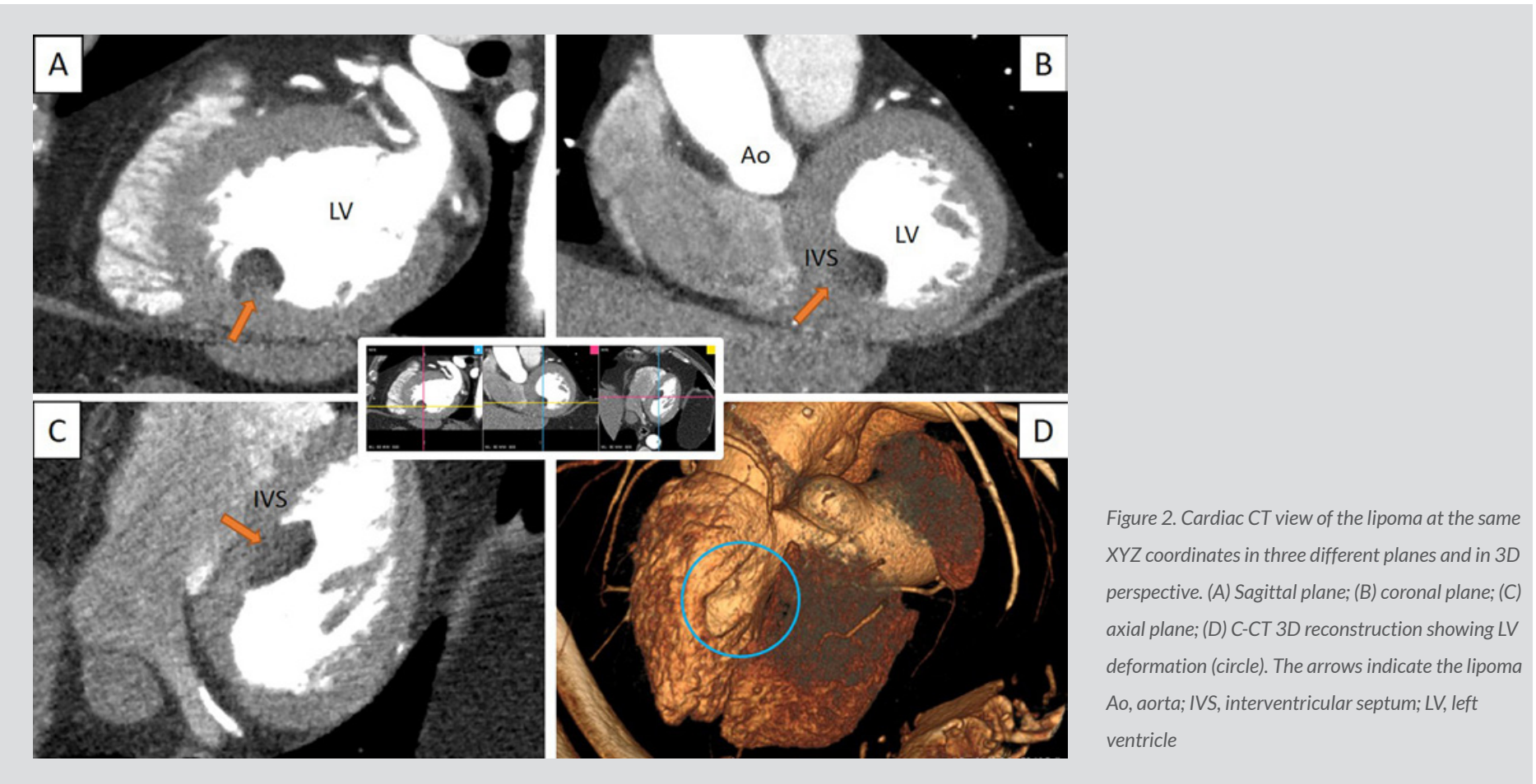

At this point it was necessary to clarify the nature of the mass and thus cardiac magnetic resonance imaging (CMRI) was performed which demonstrated an intracavitary mass implanted in the sub-endocardium, near the postero-median papillary muscle, protruding into and deforming the left ventricular cavity, with a well-defined contour. The mass showed signal suppression in fat saturation sequences, which was highly consistent with a lipoma (Fig. 3). 

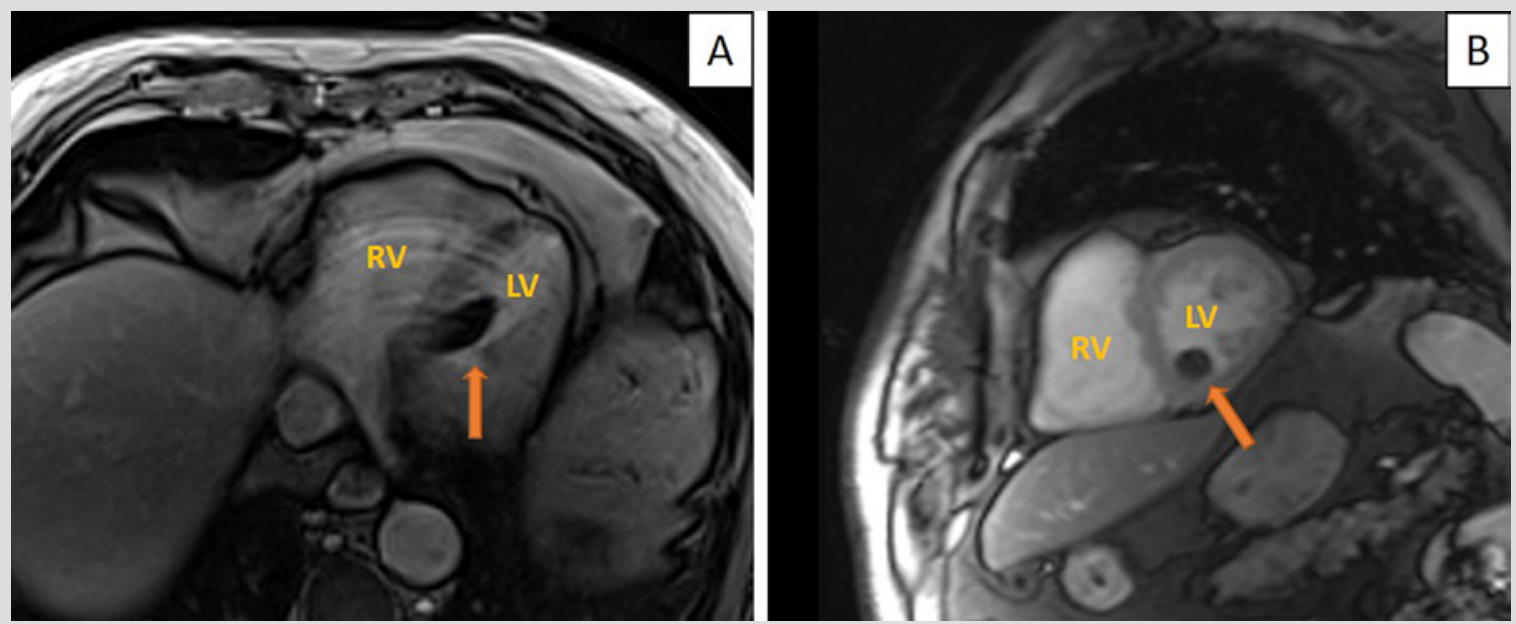

Figure 3. Cardiac MRI. (A)

Axial plane in fat suppression sequence demonstrating the IVS as a dark mass measuring $20 \times 20 \times 13 \mathrm{~mm}$; (B) sagittal plane. The arrows indicate the lipoma

$L V$, left ventricle; $R V$, right ventriclehyperactivity

The clinical picture remained unchanged in the first 2 weeks, and after cardiac surgery consultation, it was decided to adopt a conservative approach and not perform surgery or take a biopsy sample because of the radiological features consistent with a lipoma, the absence of cardiac function impairment and no recurrence of the symptoms.

The patient was discharged, with monthly evaluation in the following 6 months. The patient has remained asymptomatic during 4-year follow-up and the lipoma characteristics are unchanged.

\section{DISCUSSION}

Primary cardiac tumours are extremely rare, whereas metastatic involvement of the heart is 20-40 times more common, with sarcomas accounting for $95 \%$ of all malignant cardiac tumours ${ }^{[1]} .75 \%$ of the primary tumours are benign, nearly $8 \%$ of these are cardiac lipomas, which can be divided into true lipomas, lipomatous hypertrophy of the interatrial septum (LHIS) and lipomatous infiltration.

True lipomas consist of mature adipocytes enclosed by a collagenous capsule and may cause symptoms depending on their size and location, ranging from chest pain, arrhythmias or dyspnoea to obstruction of blood flow, whereas LHIS are a non-encapsulated collection of mature adipocytes within cardiac muscle ${ }^{[2]}$.

A review by Fang et al. revealed only six cases of IVS lipomas, from 1995 to 2014, representing a mere $2.9 \%$ of all reported cardiac lipomas with the interatrial septum being as the most common location, accounting for $38.5 \%$ of cases $^{[3]}$.

Liposarcomas, owing to their lipomatous nature, should be included in the differential diagnosis of cardiac lipomas. These are a rare subtype of sarcomas responsible for only $1 \%$ of all primary malignant cardiac tumours ${ }^{[4]}$. Prognosis is poor with death occurring weeks to months after diagnosis.

Cardiac MRI, due to its better soft tissue resolution and ability to identify cardiac thrombus, has been accepted in several consensus guidelines as the main imaging modality for cardiac mass evaluation ${ }^{[5]}$.

The current literature reports low mortality and morbidity associated with surgical excision of benign cardiac masses. Although guidelines are lacking, well accepted indications for surgery are large lipomas and symptomatic patients, while a conservative approach can be adopted with small asymptomatic lipomas ${ }^{[2,4]}$.

Primary neoplasms of the heart require a thorough evaluation and multidisciplinary approach. Choosing the best imaging modalities is pivotal for decision making concerning these rare entities. With only six cases of cardiac lipomas of the IVS reported, we hope to contribute to knowledge concerning the management of such patients.

\section{REFERENCES}

1. EIBardissi AW, Dearani JA, Daly RC, Mullany CJ, Orszulak TA, Puga FJ, et al. Survival after resection of primary cardiac tumors: a 48-year experience. Circulation 2008;118(14 Suppl):S7-S15.

2. Ismail I, Al-Khafaji K, Mutyala M, Aggarwal S, Cotter W, Hakim H, et al. Cardiac lipoma. J Community Hosp Intern Med Perspect 2015;5(5):28449.

3. Fang L, He L, Chen Y, Xie M, Wang J. Infiltrating lipoma of the right ventricle involving the interventricular septum and tricuspid valve: report of a rare case and literature review. Medicine (Baltimore) 2016;95(3):e2561.

4. Uemura S, Watanabe M, Iwama H, Saito Y. Extensive primary cardiac liposarcoma with multiple functional complications. Heart 2004;90(8):e48.

5. O'Donnell DH, Abbara S, Chaithiraphan V, Yared K, Killeen RP, Cury RC, et al. Cardiac tumours: optimal cardiac MR sequences and spectrum of imaging appearances. AJR Am J Roentgenol 2009;193(2):377-387. 